Jeremy J. Ramsden and György Kiss-Haypál

\title{
On a possible limit to economic progress
}

ABSTRACT. Economic output is shown to be related to population $(N)$ and natural resources $(R)$ by a simple power law. On the basis of the exponents for $N$ and $R$, called, respectively, the "ingenuity index" $(n)$ and the "technology index" $(r)$, the regions of the world fall into three clusters: high $n$ and high $r$ (Western and Eastern Europe, South America, Australia, New Zealand, the USA and Canada); high $n$ and low $r$ (the USA, the Middle East); and low $n$ and low $r$ (Asia, Africa). Even the highest values (of $n$ ) barely exceed unity, however. $n$ was found to be wellcorrelated with other independently obtained exponents characteristic of human ingenuity, such as those governing the number of telephone lines, patents, and the diversity of occupations. The analysis of $r$ reveals that there are two kinds of capital: natural resources and technology, especially information technology. However, endogenous productivity-depressing factors appear to impose intrinsic limits on what ingenuity and technology can achieve.

Nanotechnology Perceptions 9 (2013) 71-81

Nonsubscribers purchase individual article 